\title{
Pregnancy during a sporotrichosis epidemic in Rio de Janeiro, Brazil
}

\author{
Cassio P. Ferreira *, Antônio C.F. do Valle, Dayvison F.S. Freitas, Rosani Reis, Maria C.G. Galhardo
}

Evandro Chagas Clinical Research Institute, Oswaldo Cruz Foundation (IPEC-Fiocruz), Rio de Janeiro, Brazil

\section{A R T I C L E I N F O}

Article history:

Received 11 December 2011

Received in revised form 29 January 2012

Accepted 21 February 2012

\section{Keywords:}

Brazil

Epidemic

Pregnancy

Sporotrichosis

Thermotherapy

Sporotrichosis is a subacute or chronic disease that affects animals and humans. It is caused by the dimorphic fungus Sporothrix schenckii and is the primary subcutaneous mycosis in Latin America [1,2]. Zoonotic transmission of sporotrichosis appears to be rare worldwide. An epidemic of sporotrichosis occurred in Rio de Janeiro, Brazil, from
1998 onward. Cats were found to be the main link in the epidemiological chain [1].

From 2005 through 2010, about 1000 patients were diagnosed with sporotrichosis at IPEC-Fiocruz, including 12 pregnant women. The mean age of the 12 patients was 28.3 years (range, $18-40$ years). Two clinical forms of the infection, lymphocutaneous sporotrichosis (10 cases) and fixed sporotrichosis (2 cases), were recorded. Fungal culture was positive in all cases (Fig. 1). The patients underwent monthly outpatient assessments and the time of evolution ranged from 4-12 weeks. The upper limbs were the most commonly affected body area and cat scratch or bites were cited in 11 cases, suggesting that the lesions were the putative means of transmission of the fungus. All patients were instructed to perform thermotherapy with warm compresses 3 times a day. Eight patients were cured with thermotherapy, 2 patients had to use itraconazole after delivery, and 2 patients were lost to follow up.

The causative fungus can be found in decaying vegetation, soil, moss, and wood and can infect a diversity of animals. The usual mode of transmission is traumatic cutaneous inoculation of the

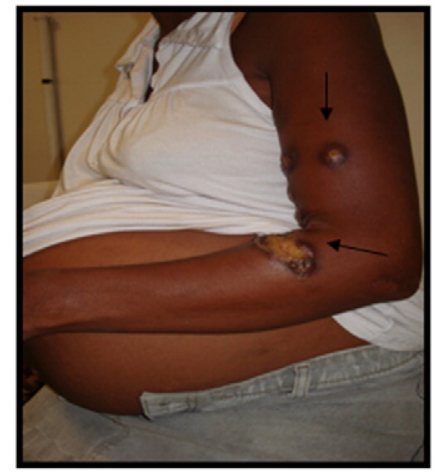

Patient 1

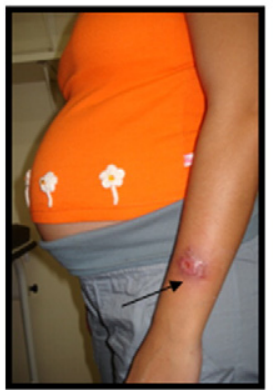

Patient 3
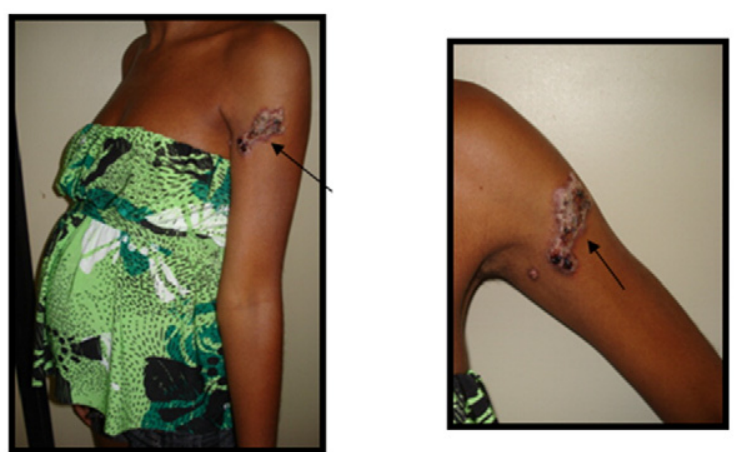

Patient 2

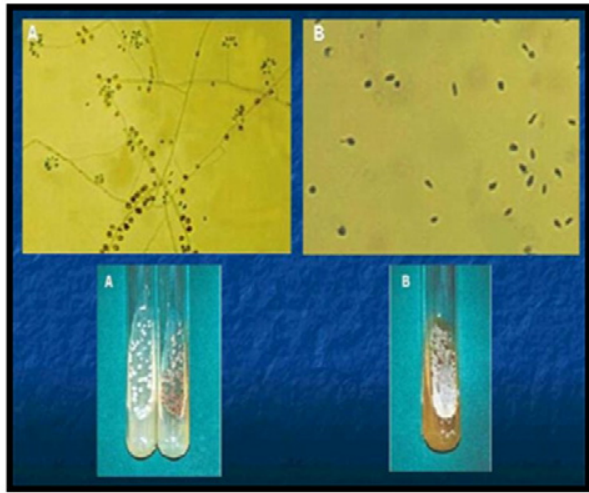

S. schenkii

Fig. 1. Cutaneous sporotrichosis in 3 pregnant patients. (A): mycelium form $\left(25^{\circ} \mathrm{C}\right)$; (B) yeast form $\left(37^{\circ} \mathrm{C}\right)$.

* Corresponding author at: Laboratório de Pesquisa Clínica em Dermatologia Infecciosa, Instituto de Pesquisa Clínica Evandro Chagas - Fiocruz. Av. Brasil 4365, Manguinhos, Rio de Janeiro, Brazil, CEP 21040-900. Tel.: +55 213865 8102; fax: +55 2122094110.

E-mail address: drcassioferreira@yahoo.com.br (C.P. Ferreira). organism [2]. Local hyperthermia can be used to treat cutaneous sporotrichosis in pregnant women [1,3]. Amphotericin B is recommended for severe sporotrichosis that must be treated during pregnancy [1]. Itraconazole is an effective fungal treatment; however, there are few data on prenatal exposure in humans and it is generally 
avoided [1]. De Santis et al. [4] reported that first-trimester itraconazole-exposed infants showed no increased risk of congenital abnormalities; however, the rates of spontaneous and induced abortions were higher in the exposed group of pregnant women compared with the control group. Various authors have reported effective treatment of sporotrichosis in pregnant women using thermotherapy $[1,3]$ and even spontaneous resolution during pregnancy [2]. Several infectious diseases, such as malaria, influenza, chickenpox, and measles, may have a markedly severe course [2] and the present cases illustrate the effective treatment of sporotrichosis in pregnancy using thermotherapy or azole therapy after delivery.

\section{References}

[1] Costa RO, Bernardes-Engemann AR, Azulay-Abulafia L, Benvenuto F, Neves Mde L, Lopes-Bezerra LM. Sporotrichosis in pregnancy: case reports of 5 patients in a zoonotic epidemic in Rio de Janeiro, Brazil. An Bras Dermatol 2011;86(5):995-8.

[2] Almeida Jr HL, Lettnin CB, Barbosa JL, Dias MC. Spontaneous resolution of zoonotic sporotrichosis during pregnancy. Rev Inst Med Trop Sao Paulo 2009;51(4):237-8.

[3] Agarwal S, Gopal K, Umesh Kumar B. Sporotrichosis in Uttarakhand (India): a report of nine cases. Int J Dermatol 2008;47(4):367-71.

[4] De Santis M, Di Gianantonio E, Cesari E, Ambrosini G, Straface G, Clementi M. Firsttrimester itraconazole exposure and pregnancy outcome: a prospective cohort study of women contacting teratology information services in Italy. Drug Saf 2009;32(3):239-44.

\title{
Conflict of interest
}

The authors have no conflicts of interest to declare.

\section{Perinatal health in rural Burkina Faso}

\author{
Judith Lindert a,b,*, Regina Breitbach ${ }^{\mathrm{a}}$, Geeke Sieben ${ }^{\mathrm{a}}$, Sontie A. Tiemasse ${ }^{\mathrm{c}}$, \\ Aboubakar Coulibaly ${ }^{\mathrm{d}}$, Jürgen Wacker ${ }^{\mathrm{a}, \mathrm{e}}$ \\ a University of Heidelberg, Heidelberg, Germany \\ b Goethe University Frankfurt, Frankfurt, Germany \\ c National School of Public Health, Ouagadougou, Burkina Faso \\ ${ }^{d}$ Regional Hospital, Dori, Burkina Faso \\ e Fürst-Stirum Klinik Bruchsal, Bruchsal, Germany
}

\section{A R T I C L E I N F O}

\section{Article history:}

Received 13 June 2011

Received in revised form 21 December 2011

Accepted 26 February 2012

\section{Keywords:}

Burkina Faso

Maternal body mass index

Perinatal health

Prenatal care

Dori in Burkina Faso is the regional capital of the Sahel region. Most people in Dori live in mud-brick houses without electricity or tap water, and rely on subsidiary farming. The majority of the population is Muslim and the dominant ethnicity is Peulh. The aim of the present study was to estimate the prevalence rate of pregnancy complications and maternal mortality at the regional hospital in Dori, and to analyze their risk factors.

The study was conducted from November 2006 to February 2008 at CHR regional hospital, Dori. Verbal consent was obtained from study participants according to national rules. Pregnant women attending the hospital for prenatal care visits or to give birth were assessed $(n=797)$; those who gave birth in the hospital where enrolled in the study $(n=452)$. The women were allocated into 2 groups: those in group 1 had received prenatal care at the regional

* Corresponding author at: Reiherstieg 4, 23564 Lübeck, Germany. Tel.: + 49451 70791351; fax: + 49725170857359 .

E-mail address: ju.lindert@web.de (J. Lindert). hospital and gave birth in the hospital; those in group 2 gave birth in the hospital. If women in group 2 had received prenatal care, this information was obtained postpartum from the mother.

Data were collected at each prenatal care visit and at delivery. The assessment consisted of 3 parts: a questionnaire before medical examination; medical assessment conducted during prenatal care visits; and birth-related variables. The questionnaire concerned sociodemographic variables and obstetric history. The sociodemographic questions concerned marital status, ethnicity, education, occupation (woman and husband), and domicile. Obstetric history was based on number of previous pregnancies and living children.

At the medical assessment the women were asked about the date of their last menstrual period (LMP), current health problems, weight, and blood pressure; a urine sample was collected and a blood test for malaria was performed. Clinical examination included signs for anemia, fundal height, palpation and auscultation of the fetus, and vaginal examination.

Blood pressure, fundal height, and maternal and fetal outcomes were assessed at delivery. Maternal morbidity was defined as postpartum hemorrhage (PPH), uterine rupture, pre-eclampsia/eclampsia, malaria during labor, and puerperal infections. PPH was classified as abnormal bleeding accordingly to the definition used in the hospital (unusual blood loss). Uterine rupture was confirmed during surgery. Pre-eclampsia was defined as a systolic blood pressure greater than $140 \mathrm{~mm} \mathrm{Hg}$ or a diastolic blood pressure greater than $90 \mathrm{~mm} \mathrm{Hg}$, plus other signs such as proteinuria, edema, severe headache, hyperreflexia, blurred vision, or oliguria/anuria [1]. Malaria during labor was diagnosed in a febrile woman in labor with a positive blood test result. Maternal mortality and mode of delivery were also recorded.

Fetal outcome was assessed by recording the number of neonates per delivery, sex, vitality, preterm birth, birth weight, and postnatal 